Please do not remove this page

RMIT

UNIVERSITY

\title{
Diffraction-managed solitons and nonlinear beam diffusion in modulated waveguide arrays
}

Szameit, Alexander; Garanovich, Ivan; Heinrich, Matthias; Minovich, Alexander; Dreisow, Felix; Sukhorukov, Andrey; Pertsch, Thomas

https://researchrepository.rmit.edu.au/esploro/outputs/9921861856801341/filesAndLinks?institution=61RMIT_INST\&index=null

Szameit, A., Garanovich, I., Heinrich, M., Minovich, A., Dreisow, F., Sukhorukov, A., Pertsch, T., Neshev, D., Nolte, S., Krolikowski, W., Tunnermann, A., Mitchell, A., \& Kivshar, Y. (2008). Diffraction-managed solitons and nonlinear beam diffusion in modulated waveguide arrays. Proceedings of the Joint 2008 Conference on Lasers and Electro-Optics 2008 and 2008 Conference on Quantum Electronics and Laser Science (CLEO/QELS 2008), 1-2.

https://researchrepository.rmit.edu.au/discovery/fulldisplay/alma9921861856801341/61RMIT_INST:Resea rchRepository

Repository homepage: https://researchrepository.rmit.edu.au

(C2008 IEEE. Personal use of this material is permitted. However, permission to reprint/republish this material for advertising or promotional purposes or for creating new collective works for resale or redistribution to servers or lists, or to reuse any copyrighted component of this work in other works must be obtained from the IEEE.

Downloaded On 2023/04/26 23:47:16 +1000 


\title{
Diffraction-managed solitons and nonlinear beam diffusion in modulated waveguide arrays
}

\author{
Alexander Szameit ${ }^{1, *}$, Ivan L. Garanovich ${ }^{2}$, Matthias Heinrich ${ }^{1}$, Alexander Minovich ${ }^{2}$, \\ Felix Dreisow ${ }^{1}$, Andrey A. Sukhorukov ${ }^{2}$, Thomas Pertsch ${ }^{1}$, Dragomir N. Neshev ${ }^{2}$, Stefan Nolte ${ }^{1}$, \\ Wieslaw Krolikowski ${ }^{2}$, Andreas Tünnermann ${ }^{1}$, Arnan Mitchell ${ }^{3}$, and Yuri S. Kivshar ${ }^{2}$ \\ ${ }^{1}$ Institute of Applied Physics, Friedrich-Schiller-University Jena, Max-Wien-Platz 1, 07743 Jena, Germany \\ ${ }^{2}$ Centre for Ultra-high bandwidth Devices for Optical Systems (CUDOS), \\ Nonlinear Physics Centre and Laser Physics Centre, \\ Research School of Physical Sciences and Engineering, \\ Australian National University, Canberra, ACT 0200, Australia \\ ${ }^{3}$ School of Electrical and Computer Engineering, RMIT University, Melbourne, Australia \\ *Phone +493641 947822; Fax +493641 947802; Email: szameit@iap.uni-jena.de
}

We present the first experimental observation of nonlinear beam diffusion and formation of diffraction-managed solitons in periodically-curved arrays of coupled optical waveguides created using femtosecond laser writing in silica glass, and titanium indiffusion in $\mathrm{LiNbO}_{3}$ crystals.

(C) 2007 Optical Society of America

OCIS codes: (190.5940) Self-action effects; (350.3390) Laser materials processing

Propagation of light in dielectric media with a periodically-varying refractive index is known to demonstrate many novel features in both linear and nonlinear regimes ${ }^{1}$. In particular, the idea to control the light spreading through diffraction management ${ }^{2}$ has attracted a special attention, and it was shown that both magnitude and sign of the beam diffraction can be controlled in periodic photonic structures. Effective engineering of light diffraction has been recently demonstrated in photonic lattices created with arrays of periodically curved optical waveguides [see, e.g., the structure sketched in Fig. 1(f)], where the self-collimation of light beams has been realized experimentally ${ }^{3,4}$. Also, the concept of the broadband diffraction management of polychromatic light has been introduced for modulated photonic lattices with special waveguide bending profiles ${ }^{5}$.
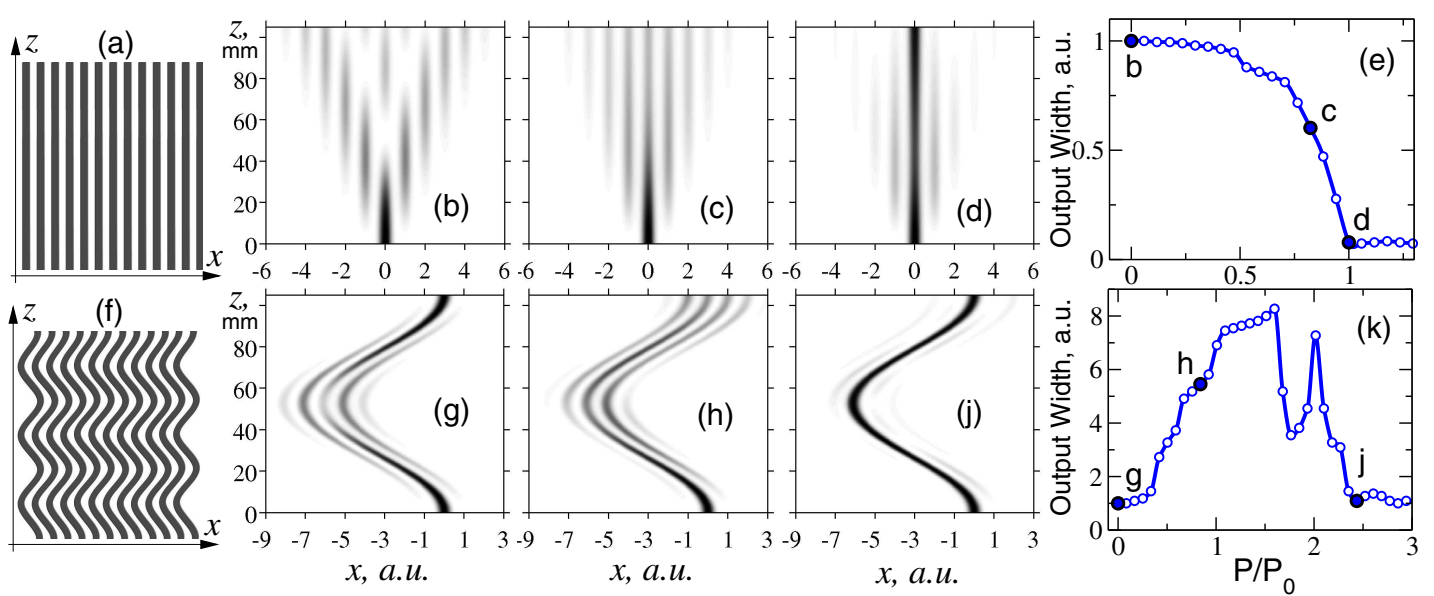

Fig. 1. Simulations of beam propagation in straight [top] and periodically-curved [bottom] nonlinear waveguide arrays. (b,c,d) Discrete diffraction, beam self-focusing, and lattice soliton in (a) straight array. (g,h,j) Self-collimation, nonlinear diffusion, and diffraction-managed soliton in ( $\mathrm{f}$ ) curved array. (e,k) Output beam width vs. the input power for straight and curved arrays. Points 'b', 'c', 'd' and 'g', 'h', 'j' correspond to the input powers in (b), (c), (d) and $(\mathrm{g}),(\mathrm{h}),(\mathrm{j})$, respectively. Coupling strength and propagation distance are the same as in Fig. 2(a).

The combination of tailored diffraction characteristics and light self-action opens new possibilities for the powercontrolled beam shaping and switching in nonlinear photonic structures. Various schemes for active beam control based on discrete spatial solitons have been suggested and demonstrated, and it was shown that solitons can also exist in diffraction-managed photonic lattices ${ }^{6}$. Recently, self-action of light beams in diffraction-managed periodically curved nonlinear waveguide arrays has been analyzed, and novel dynamical regimes have been identified depending on the input power ${ }^{7}$. In particular, it was predicted that in the periodically curved waveguide array with canceled diffraction transition from the regime of self-collimation, at low powers, to that of the discrete self-trapping and 


\section{CFR4.pdf}

formation of the diffraction-managed lattice soliton, at high powers, should occur through the intermediate regime of the nonlinear beam diffusion, as shown in Fig. 1(f)-(k). This is in a sharp contrast to the monotonous beam self-focusing which takes place in straight waveguide array as the input power increases, see Fig. 1(a)-(e).

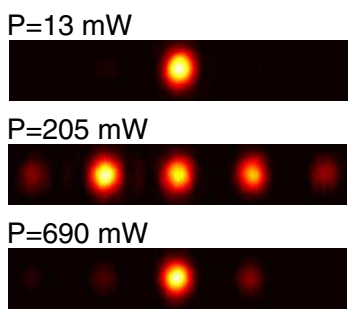

(a)

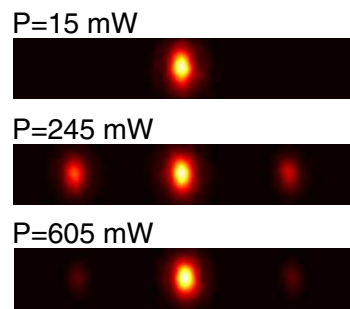

(b)

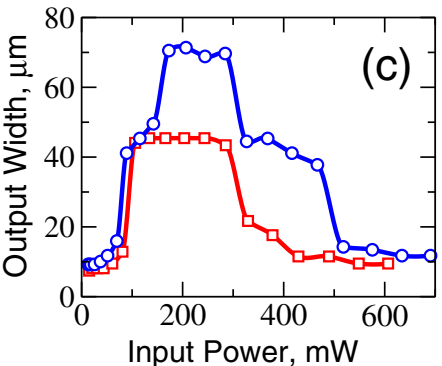

Fig. 2. (a,b) Measured output beam profiles in curved fs written waveguide arrays with lattice periods $34 \mu \mathrm{m}$ and $40 \mu \mathrm{m}$, respectively. From top to bottom: selfcollimation, nonlinear beam diffusion, and formation of the diffraction-managed soliton. (c) Output beam width vs. the input power. Circles correspond to (a), squares correspond to (b). Wavelength is $\lambda=800 \mathrm{~nm}$.

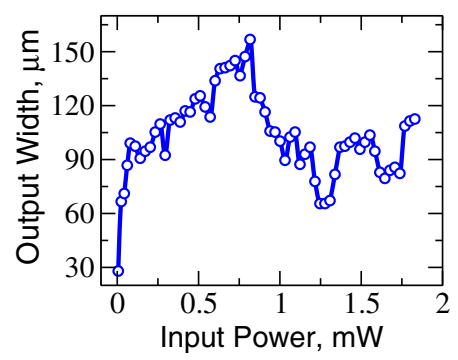

Fig. 3. Output beam width vs. the input power in $\mathrm{LiNbO}_{3}$ curved waveguide array measured at the wavelength $\lambda=$ $532 \mathrm{~nm}$.

In this work, we present the first experimental observation of nonlinear beam diffusion and formation of diffractionmanaged solitons in periodically-curved arrays of coupled optical waveguides. We have fabricated curved waveguide arrays in fused silica using the femtosecond laser writing technique. Details of the writing procedure, which was performed using a Mira/RegA (Coherent) system can be found elsewhere ${ }^{8}$. We created waveguide arrays with sinusoidal axis bending of the form $x_{0}(z)=A\{\cos [2 \pi z / L]-1\}$, in which periodic beam self-collimation takes place when the bending amplitude $A$ is such that $2 \pi \omega A / L=\xi$, where $\omega$ is the normalized frequency, $L$ is the bending period, and $\xi \simeq 2.40$ is the first root of the Bessel function $\mathrm{J}_{0} 7$. In our experiments, we used four different samples with waveguide spacing $34 \mu \mathrm{m}, 36 \mu \mathrm{m}, 38 \mu \mathrm{m}$ and $40 \mu \mathrm{m}$, and bending amplitudes $104 \mu \mathrm{m}, 98 \mu \mathrm{m}, 93 \mu \mathrm{m}$, and $88 \mu \mathrm{m}$, respectively. Each sample consisted of 13 waveguides and was $105 \mathrm{~mm}$ long.

At low laser powers, in the linear propagation regime, we observed dynamical self-collimation of light in our curved waveguides [see Figs. 2(a) and (b), top], similar to earlier experiments ${ }^{3}$. When the power of the input beam was increased, we observed nonlinear beam diffusion in the excellent agreement with the theoretical predictions ${ }^{7}$. The beam experienced significant broadening and self-defocusing [see Figs. 2(a) and (b), center] as nonlinearity destroyed the self-collimation condition by changing the refractive index of the waveguide material. At high input powers, nonlinear self-trapping of the beam eventually occurred, what we believe to be the first ever experimental observation of diffraction-managed solitons [see Figs. 2(a) and (b), bottom]. Furthermore, we have also fabricated straight counterparts for all our curved arrays and observed, in good agreement with the theory, that the critical power required for the formation of diffraction-managed solitons in curved waveguide arrays is several times higher than the critical soliton power in straight arrays. In Fig. 2(c), one can see that the critical power is lower for the waveguides with higher waveguide spacing, as the coupling decreases with the spacing increasing. Also, in Fig. 2(c) one can see that this decrease of the coupling results in less beam broadening in the regime of the nonlinear diffusion for the waveguide arrays with higher waveguide spacing.

We have also fabricated such curved waveguide arrays in a X-cut $55 \mathrm{~mm}$ long mono-crystal $\mathrm{LiNbO}_{3}$ by titanium indiffusion, and studied nonlinear beam propagation in the arrays with the defocusing nonlinearity. Similar to the case of self-focusing nonlinearity in glass, we have also observed that as the input power increases, the self-collimation regime is destroyed, and it is followed by the nonlinear beam diffusion and then by the nonlinear self-trapping at high powers, see an example in Fig. 3 for the array with the waveguide spacing $14 \mu \mathrm{m}$ and the bending amplitude $24.5 \mu m$.

In conclusion, we have observed experimentally, for the first time, nonlinear beam diffusion and formation of diffraction-managed solitons in periodically-curved arrays of coupled optical waveguides for both self-focusing and defocusing nonlinearities. These regimes are fundamentally different from the nonlinear beam self-focusing or selfdefocusing in a bulk medium, or discrete self-trapping of light in arrays of straight waveguides.

\section{References}

1. D. N. Christodoulides, F. Lederer, and Y. Silberberg, Nature 424, 817 (2003).

2. H. S. Eisenberg, Y. Silberberg, R. Morandotti, and J. S. Aitchison, Phys. Rev. Lett. 85, 1863 (2000).

3. S. Longhi, M. Marangoni, M. Lobino, R. Ramponi, P. Laporta, E. Cianci, and V. Foglietti, Phys. Rev. Lett. 96, 243901 (2006).

4. R. Iyer, J. S. Aitchison, J. Wan, M. M. Dignam, C. M. de Sterke, Opt. Express 15, 3212 (2007).

5. I. L. Garanovich, A. A. Sukhorukov, and Yu. S. Kivshar, Phys. Rev. E 74, 066609 (2006).

6. M. J. Ablowitz and Z. H. Musslimani, Phys. Rev. Lett. 87, 254102 (2001).

7. I. L. Garanovich, A. A. Sukhorukov, and Yu. S. Kivshar, Opt. Express 15, 9547 (2007).

8. A. Szameit, F. Dreisow, T. Pertsch, S. Nolte, and A. Tunnermann, Opt. Express 15, 1579 (2007). 